\title{
Heritage and Advanced Technology Systems Engineering Lessons Learned from NASA Deep Space Missions
}

\author{
Bryan Barley ${ }^{*}$, Allen Bacskay ${ }^{\dagger}$, \\ National Aeronautics and Space Administration, George C. Marshall Space Flight Center, \\ Marshall Space Flight Center, Alabama, 35812, USA \\ Marilyn E. Newhouse ${ }^{\ddagger}$ \\ CSC \\ Marshall Space Flight Center, Alabama, 35812, USA
}

\begin{abstract}
In the design and development of complex spacecraft missions, project teams frequently assume the use of advanced technology systems or heritage systems to enable a mission or reduce the overall mission risk and cost. As projects proceed through the development life cycle, increasingly detailed knowledge of the advanced and heritage systems within the spacecraft and mission environment identifies unanticipated technical issues. Resolving these issues often results in cost overruns and schedule impacts. The National Aeronautics and Space Administration (NASA) Discovery \& New Frontiers (D\&NF) Program Office at Marshall Space Flight Center (MSFC) recently studied cost overruns and schedule delays for 5 missions. The goal was to identify the underlying causes for the overruns and delays, and to develop practical mitigations to assist the D\&NF projects in identifying potential risks and controlling the associated impacts to proposed mission costs and schedules. The study found that optimistic hardware/software inheritance and technology readiness assumptions caused cost and schedule growth for four of the five missions studied. The cost and schedule growth was not found to result from technical hurdles requiring significant technology development. The projects' institutional inheritance and technology readiness processes appear to adequately assess technology viability and prevent technical issues from impacting the final mission success. However, the processes do not appear to identify critical issues early enough in the design cycle to ensure project schedules and estimated costs address the inherent risks. In general, the overruns were traceable to: an inadequate understanding of the heritage system's behavior within the proposed spacecraft design and mission environment; an insufficient level of development experience with the heritage system; or an inadequate scoping of the system-wide impacts necessary to implement an advanced technology for space flight applications. The paper summarizes the study's lessons learned in more detail and offers suggestions for improving the project's ability to identify and manage the technology and heritage risks inherent in the design solution.
\end{abstract}

\section{Introduction}

The Discovery Program (DP) is a science program of frequent, mid-class spacecraft missions that perform highquality focused scientific investigations. Initiated in 1992, the DP was defined to ensure frequent access to space for planetary system(s) science investigations, emphasizing missions that can be accomplished under the leadership of the scientific research community. The DP comprises a long-term series of space science missions that are independent and uncoupled, but share a common funding and management structure. Since its inception, DP has successfully completed missions to study the Moon, inner planets, asteroids, comets, and solar wind. Current missions in development or operations will continue exploration of the inner and outer planets, asteroids, comets, and the Moon.

\footnotetext{
* Lunar Quest Program Chief Engineer, MSFC/EE04.

${ }^{\dagger}$ Discovery and New Frontiers Deputy Program Manager, MSFC/VP23.

${ }^{\ddagger}$ Principal Lead Systems Engineer; MSFC/VP23.
} 
The New Frontiers Program (NFP) is a science program of medium-size spacecraft missions that perform highquality focused scientific investigations. Initiated in 2003, the NFP was defined to pursue planetary missions that require resources beyond those available in the DP. The NFP comprises a long-term series of space science missions that are independent and uncoupled, but share a common funding and management structure. The NFP currently includes two missions to study outer and dwarf planets.

Missions for both programs are selected through an open science competition and can include any science investigation involving solar system objects except for the Earth and the Sun, which are currently covered by other programs. D\&NF missions are Principal Investigator (PI)-led, complete, self-standing, and uncoupled Science Mission Directorate (SMD) investigations. The total cost to the National Aeronautics and Space Administration (NASA) for each full mission has a not-to-exceed cost cap specified in the Announcement of Opportunity (AO) for the competition. At the time of this study, the Discovery mission cost cap was $\$ 425 \mathrm{M}$ and the New Frontiers mission cost cap was $\$ 700 \mathrm{M}$. The mission cost cap covers the complete mission, including spacecraft development, mission operations, data analysis, and education and public outreach.

D\&NF missions are ultimately defined in terms of the science return from the mission. Level I requirements include the baseline science mission: the full set of scientific requirements identified for the mission, and the threshold science mission: the minimum set of science requirements below which the mission is not considered justifiable for the proposed cost. Each PI is held responsible for proper execution of all aspects of the mission, including implementation and execution within the confirmed mission cost and schedule.

The D\&NF programs are managed by a single program office at Marshall Space Flight Center. As uncoupled, multi-mission programs emphasizing cost-capped PI-led missions, the ability of the D\&NF programs to meet their launch frequency requirements is driven by the ability of each individual project to meet its proposed and confirmed life cycle cost (LCC) and schedule. Looking at the missions over the history of the D\&NF programs showed an increased frequency of cost overruns (see Figure 1).

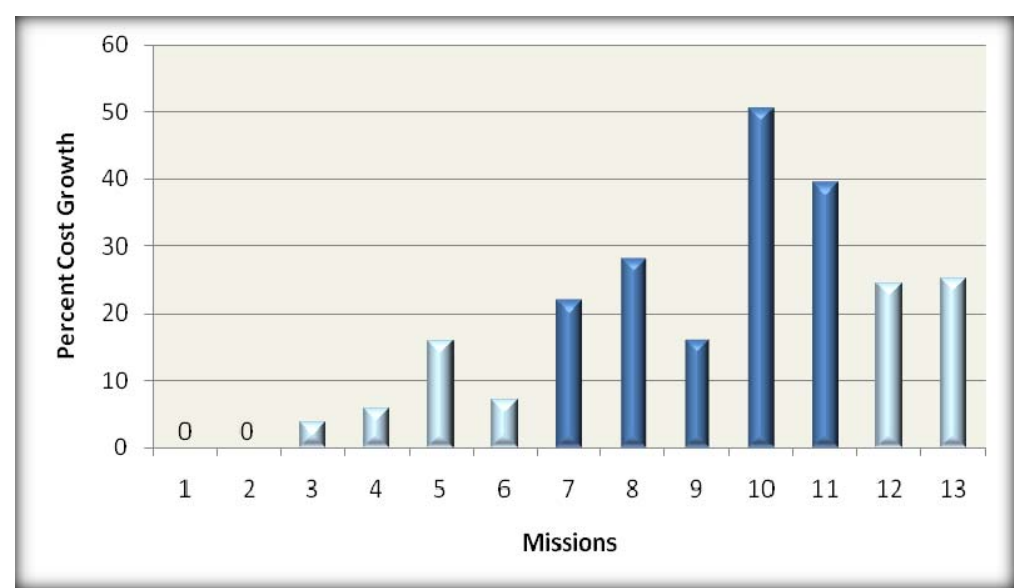

Figure 1. D\&NF Life Cycle Cost Growth

While there was a trend of increasing life cycle cost growth even in the earliest D\&NF missions, the growth was contained in the early missions, showing an average cost growth of $\sim 2 \%$ and a maximum of $15 \%$ for one mission. In contrast, the five recent missions selected for the study showed an average cost growth of $31 \%$, with one mission reaching 50\%. This growth directly affects the programs' ability to fund new mission starts and meet their objectives to provide opportunities for the science community to propose and execute solar system exploration missions.

Therefore, the Program Manager for D\&NF initiated a study of the cost escapes on recent D\&NF missions to identify the primary drivers and determine what reasonable things could be done as a program to either prevent the cost escapes or manage them better. Five missions were selected from the two programs based on a recent history of exceeding proposed or confirmed costs. Those missions covered a spectrum of complexity, cost growth, and maturity and are highlighted in dark blue in Figure 1. The study process and results are detailed in the final report, "Improving the Life Cycle Cost Management of Planetary Missions.”

\section{Heritage and New Technology in D\&NF Missions}

The study found that among other cost drivers, four of the five missions experienced cost growth that could be traced to issues involving the use of heritage or new technology. The study also indicated that the cost growth could be traced to problems that were embedded as a result of decisions that were made during formulation (phases $\mathrm{A}$ and B), even if the costs were not realized until late in phases $C$ and D. This paper expands the original life cycle cost study and provides a more detailed look specifically at the cost growth drivers experienced during the inheritance of technology or the use of new technology for six recent D\&NF missions. This paper concentrates on the technology drivers affecting development and integration (phase C \& D) costs. Additional implications of the use of heritage 
hardware and software for the growth in operations (phase E) costs are addressed in a separate paper, "Planning and Estimation of Operations Support Requirements.” A limited discussion of the effect of heritage technology on fault management systems can be found in "Results from the NASA Spacecraft Fault Management Workshop: Cost Drivers for Deep Space Missions.”

As small to medium, low cost, deep space missions, the D\&NF programs specifically emphasize mature technologies with a high technology readiness level (TRL). Thus, mission proposals typically emphasize the inheritance of heritage hardware or software from previous missions, and cite the heritage systems as justification for lower development and operations cost or decreased mission risk. However, the TRL of a given subsystem or component is separate from its heritage or the process of inheriting (incorporating) the system or component into the mission system.

Less frequently, D\&NF mission proposals also specify the use of new (low TRL) technology to enable a specific mission capability. In these cases, the proposals include the steps the project will undertake to qualify the new technology, which often include synergy with or reliance on other project's qualification activities to lower the D\&NF project cost or risk.

For the purpose of this paper, heritage technology includes hardware or software subsystems or components with previous flight history that are used as part of a new mission system. The heritage of the component includes not only the previous flight history, but the previous function(s) for which the component was used, the environment in which it was used, and physical, thermal, and data interfaces with other elements of the mission system. Heritage also includes the availability of documentation, support equipment, and personnel experienced in its design, implementation, and operational use.

Inheritance covers the system engineering and development processes for incorporating the technology into the mission system. Inheritance includes evaluation and review of the level of heritage applicable for a component, determination of the level of reuse, redesign, or rebuild necessary for the technology to execute the required functions as a part of the new mission system, and development of the constraints, procedures, and products required to execute the mission using the heritage technology.

Technology readiness level is a measure of the maturity of a given technology. Typically, high TRL components, prototypes, and models have been proven to operate as expected, usually in space. Thus, heritage technology is by definition at a high TRL. However, as will be discussed more fully below, a high TRL does not ensure that there will be no changes either to the heritage technology to accommodate the current mission needs, or to other components, particularly when multiple heritage technologies (e.g., a spacecraft bus and avionics or science instruments) are combined into a single mission system.

\section{Cost Growth Drivers}

For most of the D\&NF missions studied, institutional inheritance processes for the individual projects included detailed subsystem-level reviews during formulation focusing on the applicability of the proposed heritage technology to the mission requirements. The reviews considered the previous flight history of the technology, the applicability of the heritage technology to the current mission and environment, required changes to its design or operational use, and the schedule and staffing for any changes required to use it within the spacecraft and mission environment. This process worked well to identify technical issues associated with the selection and use of heritage technology. Although there were no institutional reviews specifically aimed at new technology, the standard institutional process for detailed peer reviews of each subsystem prior to PDR and CDR served well to catch technical issues relating to the new technology. However, for both heritage and new technology, by the time the technical issues were identified and addressed, the corrections to or mitigation of the issues resulted in schedule delays and cost increases.

In one form or other, the schedule delays and cost impacts could be traced back to overly optimistic assumptions about the time and effort required to incorporate heritage hardware into or to certify new technology for use within the missions system. These assumptions included inadequate consideration of the differences in spacecraft configurations and operational environments between the heritage technology and the proposed mission system, overly optimistic assumptions about availability of expert personnel, unjustified decrease in project insight/oversight of heritage system design and development, the assumption of heritage and inheritance with minimum cost without proven in-flight performance, inexperience with a new technology, late design changes, and overreliance on synergy with other projects for new technology certification. These drivers will be discussed in more detail in the following sections. 


\section{A. Insufficient Concept Development/Validation during Formulation}

One of the primary causes of cost and schedule growth associated with heritage hardware was inadequate consideration of changes to the spacecraft configuration and the operational environments of the heritage technology compared to the mission being proposed. Projects often take the simplistic approach during early formulation that a piece of hardware has flown before and therefore has been proven to work, without taking into account the environments in which the technology was flown in the past and will be flown in the new mission. This environment can include the spacecraft systems environment (e.g., loads, power, thermal, contamination, etc.) or the mission environment (e.g., thermal, radiation, data rates, time delays, expected life time, etc.). As the detailed design process uncovers issues with the initial assumptions, the project may need to redesign some or all of the heritage technology, as well as other mission systems.

For example, thermal analysis may show that temperatures will be higher than operational constraints for a heritage component as located on the spacecraft, requiring additional thermal protection, relocating the heritage component, or replacing the heritage component with another option. Alternatively, thermal analysis may show that the heritage component will generate too much heat in the mission system for nearby components, again requiring additional thermal protection, relocation, or possibly replacement. As another example, a science instrument that has flown successfully on multiple low Earth missions, may require additional radiation hardening or radiation shielding for use in a deep space environment. These changes may result in a significantly large increase in mass that other spacecraft elements must be redesigned to reduce overall system mass. In either case, the resulting components look less and less like the original heritage items, typically without having undergone the same rigorous review process as for new development.

Not only do these issues increase the overall time and cost for design and redesign, but identification of significant changes late in the formulation process (phase B or early phase C) may not allow adequate time for long lead procurements identified as mitigations. Alternatively, when multiple heritage technologies are selected for a single mission, the biggest threat to mission cost and schedules may not be a major redesign to a single component, but a continuous stream of multiple, small changes to the overall mission system design. The project team ends up chasing a stable configuration, detracting from its ability to perform true systems analyses to validate the mission. Finally, as the number of modifications to the initial design increase, schedule pressures limit the consideration of related changes to integration procedures, test plans, and operations procedures or products; substantial integration and test workarounds; or some level of regression retesting. All of these result in additional cost increases, or possibly schedule delays, late in the development cycle.

One D\&NF mission assumed the use of a previously flown science instrument to measure the same science parameters, but in a different planetary environment and under a different science operations concept. Subsequent analyses of the expected environment at the planetary target identified significant modifications to the instrument design to allow it to operate in a higher radiation environment, and required the use of different science models to produce useful, calibrated data and meet the mission science requirements. In this case, the project was able to meet the development schedule milestones, but only by using all available instrument development schedule reserve and almost all science instrument cost reserves to address the technical issues resulting from the change in operational environment.

Another D\&NF mission studied proposed a science instrument that shared a number of design concepts with, and was estimated to take advantage of thermal design synergies with, a similar instrument on another spacecraft in development. Thermal analyses performed during development identified a number of thermal issues resulting from the mission's multi-instrument configuration. These issues required redesign of the multi-instrument configuration, resulting in the loss of thermal design synergy and significant cost increases to the project in development (phases $\mathrm{C}$ and D).

\section{B. Premature Claims of Proven Heritage}

Projects also claim heritage for technology that has not flown or has not operated for its full planned lifetime. Proposed missions will include science instruments, flight software, or other components that are nearing operations (launch) or are in-flight to the final science destination, and claim reduced development costs or time based on the heritage of the technology. The reduction in cost or development time is then not realized when in flight anomalies require analysis support or redesign, or when in-flight performance is not as expected.

One mission studied assumed heritage for a suite of science instruments as a risk and cost reduction for instruments that had been or were being used on several missions. Because the instruments were in flight and operating nominally, the project did not fully validate the science instruments for use at the planned science target and environment. Subsequent science data analysis identified that modifications to the selected suite of instruments would be required to ensure that the mission science could be obtained at the planned destination. This was

4

American Institute of Aeronautics and Astronautics 
identified in time to make the changes to the instrument designs prior to launch, however launch was delayed (although not solely due the instrument design changes) and additional funding was required to cover the instrument modifications.

Similarly, during phase A for another D\&NF project, trade studies included a command system that was being flown on a recently launched mission and also being used on another project in development. Each of the three projects was developed by the same mission design institution and its external prime development organization; the inheritance process was disciplined and thorough and had been used many times. Institutionally therefore, the inheritance processes appeared to address technical viability to prevent technical issues from impacting final mission success. However, after the D\&NF project entered the critical design phase (phase $\mathrm{C}$ ), the in-flight project experienced several unexplained anomalies. Both projects still in development had to assign engineers to the flight project investigation team to ensure that the development projects clearly understood the root cause of the anomalies, the applicability of the root cause to their mission systems, any hardware, software, or operational modifications that might be required or desired based on the root cause, or the downstream effects on their systems if a root-cause could not be determined. In the event that fixes could not be implemented pre-launch, the project teams needed to be in a position to plan for interchanging or regression testing engineering and flight units during spacecraft integration and test (phase D) and to recommend in-flight operational workarounds to mitigate the risk of anomalies. All of this support created an unanticipated drain on project development personnel, delaying planned work and increasing development costs.

\section{Overly Optimistic Estimates of Effort}

Even when the mission environment and operations concept has been thoroughly considered, the heritage of the technology is directly relevant and proven, and all or most of the required changes to the heritage technology have been identified, the missions studied showed that projects still tend to be overly optimistic about the effort that will be required to incorporate heritage technology or qualify new technology. Manufacturing processes and the availability of materials change, requiring revalidation and qualification for the planned operational environment and life time. Expert personnel may not be available when required to support project teams. These, and other similar issues, are not considered and adequately mitigated during project planning.

As one example from the cases studied, a D\&NF project proposed a high level of heritage for a propulsion system on system flown several years earlier on a successful NASA mission without properly verifying the heritage assumptions during formulation (phases A and B). Due to a number of design and programmatic issues (e.g., expert personnel availability and insufficient insight/oversight, see below), the propulsion system design underwent significant changes during formulation and design (phase B and well into phase C). When cost growth threatened to cancel the mission during the design phase (phase C), an independent assessment indicated only a moderate level of inheritance could actually be claimed for the as-designed propulsion system. The difference between the initial project assumptions about the level of inheritance and the actual level of inheritance achieved drove, in large part, the growth in LCC for the mission.

Reaction wheels, which have flown successfully on hundreds of missions over the years, are one example that has affected the risk, spacecraft development schedules, and cost estimates for several of the D\&NF missions studied. Most projects would consider reaction wheels as high TRL, flight proven technology, easily incorporated (inherited) into the system design. However, several recent missions across NASA have included cases of early reaction wheel failures during mission operations and workmanship issues uncovered during integration and testing. This has required the allocation of D\&NF project resources to follow or support failure analyses, understand the implications for the current projects, and develop mitigation strategies or perform trade studies of alternatives, all at a significant cost to mission schedules and resources.

Overly optimistic estimation of effort is also an issue for the qualification of new technology required to meet the mission science requirements. One project studied proposed to integrate a considerable amount of relatively new technology into spacecraft as part of the mission concept. Formulation (phase B) analyses and the Preliminary Design Review (PDR) process did not identify any significant risks associated with the level of new technology used. However, during development (phase C), the project was faced with the considerable challenges of simultaneously qualifying advanced thermal, communications, composite materials, and propulsion tank designs. The coupling between this qualification of "cutting-edge" technology designs and the typical spacecraft development issues (mass margins, autonomy system complexity, instrument integration, etc.) was underestimated and resulted in significant cost growth during development and spacecraft integration. The cost growth was exacerbated by pressure to maintain the critical launch date for a planetary launch, and the overall mission cost and the operational complexity grew significantly when the launch had to be delayed due to the growth in effort required to qualify the new technology and test the integrated system.

5

American Institute of Aeronautics and Astronautics 
In another case studied, a D\&NF project committed to the use of critical avionics hardware, claiming heritage while the subsystem hardware was still in development for another project. The D\&NF project assumed that it would be able to take advantage of the work in progress on the other project, and defined the system engineering processes and estimated the associated effort based on an assumption of inheriting the avionics hardware when complete. The reduced systems engineering approach and the lack of engineering development units at the box level resulted in unexpected troubleshooting and significant design changes to the avionics hardware late the in the project development phase. Commitment to a critical component at a low TRL critical avionics subsystem, combined with an attempt to use a heritage approach based on the assumption of synergies with another project in development led to significant cost increases and schedule delays. Given the launch constraint requirements for the planetary target, the delays ultimately resulted in a 1-year launch slip.

Another important consideration for estimating the use of a technology is the availability of personnel who are experts in the development and use of the technology. One D\&NF project proposed the use of advanced charge couple device (CDD) and optics technologies to enable the mission science. The project initiated a targeted risk reduction effort and long lead procurement during early formation (phase A) to mitigate the risk associated with maturing the science instrument. This was a sound management approach, but the engineering team's limited expertise with the new technology limited the approach's ability to mitigate the risk to an acceptable level and the instrument ended up on the critical path to launch. External CCD/optics technology experts were brought into assist the project team, and the technical issues were resolved, but at additional cost and several schedule delays.

Engineers with in depth knowledge of the heritage technology design, the reasoning behind certain trades, the use to which and the environment in which the technology was operated, are just as critical to realizing design and development cost and schedule savings. One D\&NF project studied made overly optimistic assumptions about the availability of expert personnel, without sufficient mitigation for schedule delays when the experts were not available as required by the project schedules. The original project plan relied on the availability of personnel coming off development of another project. The project had also selected to reduce risk by increasing their use of heritage hardware and software, an approach that was put at risk when technical issues delayed launch of the earlier project and the availability of the expert personnel. The D\&NF project chose to rework their schedules to wait for the experts to become available, but the resulting delays meant that internal deliveries were late in the development and integration flow of the spacecraft, thereby increasing risk.

Finally, projects often estimate costs and plan development schedules based on an overly optimistic decrease in the level of required insight or oversight for heritage technology. Teams can assume that heritage designs are inherently more reliable than new designs, and can plan to skip process steps such as tabletop reviews for hardware schematics or inherited software designs. In one case studied, the project technical engineering team relied heavily on a critical heritage sensor circuit design. The prevalent view of the team was that because the circuit design was inherited, it was more reliable and therefore required less scrutiny than new and unproven designs. Ultimately, as a result of a design flaw missed due to the abbreviated technical reviews, the circuit failed in operations causing a system level failure. Although the science data was recoverable, the recovery was only possible after significant replanning, unexpected workarounds, and additional cost for the science data collection and processing.

\section{Mitigations}

Use of heritage and new technology within a mission system is a necessary and enabling approach to implementing small, low cost missions. No one would deny that using systems with flight proven heritage reduces mission risk and holds the potential to reduce mission cost. Likewise, it is not practical to assume that a project can complete all detailed designs, analyses, and trades prior to submitting a proposal in order to ensure that the proposed cost and schedule are realistic, and cost growth and schedule delays can be contained. However, there are steps that can be taken to limit the risk of cost growth and schedules delays resulting from the use of new or heritage technology.

First project management needs to ensure the time and resources are available to perform critical analyses early in project formulation. Project management and systems engineering should work together to identify the key risks associated with any proposed heritage or new technology. Specifically, this analysis needs to consider

- Differences between the flight (for heritage technology) or planned (for new technology) spacecraft and operational environments

- Actual in-flight experience: has the technology been flown for the full planned mission

- Realistic synergy with parallel development efforts

- Availability of expert personnel as needed by the formulation and development schedule 
- Careful tailoring of engineering processes and insight/oversight for inherited technology

- Changes in manufacturers, manufacturing processes and materials

Each risk should be clearly identified, mitigation strategies developed and tracked, and threats against the project reserves should be assigned. Engineering analyses and trades should be prioritized and scheduled based on this risk assessment. The threats against the project reserves should be reassessed regularly as the formulation and development process progresses.

To mitigate against concerns that early identification of development risks and increasingly realistic cost estimates will make specific proposals less "competitive," evaluation processes need to differentiate between project risks and project weaknesses. A well understood and mitigated risk is not necessarily a weakness; realistic assessments of the true costs of inheriting or qualifying technology should be considered a strength.

\section{Conclusion}

As small cost-capped missions, controlling mission risk and reducing mission development cost is of primary importance to the D\&NF projects and programs. As competed missions, D\&NF proposals will always drive to a lower proposed mission cost and risk in order to ensure the proposed mission is competitive and make the proposed mission more desirable (increase the ratio of the science benefit to the total mission cost and risk). Heritage technology is a strong tool for achieving these goals, but it is not without inherent risks of its own. The D\&NF experience is that missions rarely achieve the full potential for reducing mission cost and risk inherent in the use of heritage technology. Similar issues affect a project's ability to accurately estimate and control cost growth for new technologies required to enable a particular mission.

When inheritance processes are used that emphasize a detailed review of the intended use of heritage technology within the mission system, they adequately identify the technical issues in the design or operational use of the technology. This allows the project to identify and implement the necessary design or operations changes, but not without impacts to the estimated project costs and the major project milestones. The D\&NF study of the schedule delays and cost overruns related to the use of heritage technology identified several fundamental drivers: inadequate consideration of changes in the spacecraft configuration and the operational environments of the heritage technology compared to the previous flight use, overly optimistic assumptions about the cost of or time required for inheritance of the heritage technology, overly optimistic assumptions about availability of expert personnel, premature claims of heritage before in-flight performance has been proven, and an unjustified decrease in process rigor or the level of insight/oversight for heritage technology. Inadequate consideration of the spacecraft configuration and mission environment, overly optimistic assumptions about the level of qualification or synergy with other projects, and the availability of expert personnel also contributed to cost growth when using new technology in D\&NF missions.

To mitigate the inherent risks in the use of heritage technology and allow projects to take full advantage of the benefits of inheriting proven technologies, additional emphasis is required during proposal development and early project formulation on the realistic assessment of heritage technology assumptions, identification of risks to those assumptions, and development of mitigation strategies. A similar emphasis on realistic estimates of the effort required to qualify new technology, potential risks to the estimates, and appropriate mitigation strategies will help projects control the cost growth associated with the use of new technologies. Mitigation strategies should include an appropriate allocation of project reserves based on a risk assessment of the inheritance process for each individual area of heritage or new technology and careful tailoring of inheritance processes to ensure that due diligence is maintained for heritage technology.

\section{Appendix A. Glossary}

Heritage Systems: Hardware, software, and procedures with previous flight history that are reused for a new mission in order to enable a mission capability or reduce overall mission cost, schedule, or risk.

Inheritance: The process of evaluating the compatibility and benefits of heritage systems to the requirements of a new project, and validating the level of reuse or rework (design, fabrication or coding, process or procedure development, documentation) required to use the heritage system in the new mission environment.

Technology Readiness Level: A measure of the maturity of new technologies and the likelihood of its success when used operationally to achieve mission success. 


\section{Acknowledgments}

The Discovery \& New Frontiers Program Office Life Cycle Cost Study was performed under the direction of Paul Gilbert (MSFC), led by Bryan Barley (MSFC), and supported by Kenny Mitchell (MSFC-retired) and Marilyn Newhouse (CSC).

\section{References}

${ }^{1}$ Barley, B., Gilbert, P., and Newhouse, M., “Improving the Life Cycle Cost Management of Planetary Missions,” February 2010.

${ }^{2}$ Newhouse, M., Barley, B., Bacskay, A., and Clardy, D., “Planning and Estimation of Operations Support Requirements,” SpaceOps 2010 Conference, April 2010.

${ }^{3}$ Newhouse, M., McDougal, J., Barley, B., Stephens, K., and M, Fesq, L., "Results from the NASA Spacecraft Fault Management Workshop: Cost Drivers for Deep Space Missions,” SpaceOps 2010 Conference, April 2010. 